\title{
EDITORIAL
}

\section{MATERNAL HEALTH DURING PREGNANCY AND SAFE DELIVERY}

\author{
DR. IFFAT ARA
}

Associate Professor, Department of Obstetrics \& Gynaecology, Dhaka Medical College \& Hospital, Dhaka

J Dhaka Med Coll. 2010; 19(1) : 1.

Childbirth is a universally celebrated event, an occasion for merry making. Yet for many thousands of women childbirth is experienced as a private hell that may end in death. In fact, maternal death and injury in developing countries constitute a tragedy of vast proportions, yet it is a tragedy that has been largely ignored by those who set national and international health priorities because those who suffer generally live in remote places, are poor illiterate and politically powerless.

The Fifth Millennium Development Goal (MDG) target for maternal survival is to reduce maternal mortality by two-thirds by 2015 . Given that the maternal mortality rate is at present 3.2 per thousand live births. In low income countries over the last decade there has been no measurable reduction in maternal mortality. The solution to reduce maternal deaths is intimately linked to maternal health and to provision of effective maternal health services with special emphasis to safe delivery. Thus, addressing current global gaps for care at birth is critical to achieving MDG 5, for maternal mortality reduction.

The vast majority of maternal deaths are clustered in and round intrapartum and postpartum period. Most of the direct obstetric causes of maternal deaths are due to obstetric hemorrhage, puerperal sepsis, hypertensive complications and prolonged/ obstructed labor. Therefore the time of greatest risk of mortality is at birth. This underscores the urgent need to coordinate childbirth and early postnatal interventions for both the mother and the baby.

Maternal death has many causes and it therefore requires actions on several fronts simultaneously to combat it. The need for improvement in the social status of women is an important step forward. Other interventions are healthcare before pregnancy, throughout pregnancy, safe delivery by skilled birth attendant and postnatal care.

Health care during pregnancy and safe delivery: Potentially one of the most effective health interventions for the prevention of maternal morbidity and mortality is prenatal care. It has several major functions:

(a) The promotion of health during pregnancy through advice and activities.

(b) The screening, identification and referral if necessary with risk factors.

(c) The monitoring of health throughout pregnancy and to be aware of the danger signs of pregnancy like blurring of vision, headache, bleeding, malpresentation and early rupture of membrane.

(d) Birth preparedness

(e) Skilled birth attendance at delivery \& provision of emergency obstetric care

(f) Early referral \& linkage in case of emergency

In conclusion, maternal health in pregnancy $\&$ safe delivery should be a continuum of care approach. This is a conceptual framework for integrated

Maternal, neonatal and child health. Programs must address risk factors across the entire continuum of life cycle from adolescence through pregnancy and childbirth. 